\title{
Editorial
}

\section{Effective haemostasis during cardiac surgery}

Excessive bleeding following cardiopulmonary bypass (CBP) remains one of the major potential problems in cardiac surgery. In addition to inadequate surgical haemostasis, pre-existing disease, heparin-protamine interaction, platelet quantity/quality deficiency, fibrinolysis, and malignant hypertension are the other major potential causes of excessive bleeding. In this issue Hardy and Desroches review the usefulness of natural and synthetic antifibrinolytics in cardiac surgery. ${ }^{1}$ While pharmacological intervention with these agents has been shown to decrease blood loss and, in some cases, homologous blood requirements, other methods or pharmacological agents are available.

The best way to detect a haemorrhagic diathesis is a properly taken history. The haemostatic response to any previous surgery and current medications (e.g., aspirin, which inhibits platelet function at low doses) may suggest a need for additional tests. Furthermore, in major cases where bleeding is a potential problem a routine haemostatic screening profile is helpful in ruling out a pre-existing lesion when excessive bleeding ensues intraoperatively. With a negative history and a normal profile the absence of a pre-existing haemorrhagic diathesis is a legitimate assumption.

Except for the use of ancrod to defibrinate the patient over a 24-hr period before surgery, a technique not likely to gain widespread acceptance due to the long preparation time preoperatively and the absolute need to administer an homologous source of fibrinogen post- cardiopulmonary bypass, all patients who require use of the heart-lung machine must receive heparin prior to bypass and will require an antidote, usually protamine, post-bypass to neutralize the heparin. Residual heparin and excessive protamine have both been implicated as causes of excessive bleeding.

Bull et al. demonstrated a threefold variation in sensitivity and a fourfold variation in duration for a given dose of heparin in a large patient population, indicating that there

From the Hahnemann University, ${ }^{*}$ Philadelphia, PA and the University of Pennsylvania, $†$ Philadelphia, PA may be as much as a twelvefold variation in individual patient response to a given dose of heparin. ${ }^{2}$ They suggested borderline or inadequate anticoagulation during cardiopulmonary bypass may result in low-grade activation of coagulation, resulting in depletion of coagulation factors and secondary fibrinolysis, and advocated heparin titration to insure adequate anticoagulation prior to cardiopulmonary bypass. The dramatic decrease in the incidence of extensive fibrinolysis following cardiopulmonary bypass seen the in late 1970 s may be explained by more complete anticoagulation resulting from almost universal acceptance of the heparin titration concept. ${ }^{3}$

With rare exception, protamine is the only antidote used to neutralize heparin. Many heparin-protamine ratios have been proposed for neutralizing heparin. However, confirmation of neutralization is essential and a protamine titration is an easy way to demonstrate the presence or absence of heparin conclusively.

Avoidance of malignant hypertension does not mean deliberate hypotension. Rather, the provision of adequate anaesthesia combined with appropriate vasodilators or beta blockers to avoid the stress on suture lines with potentially catastrophic results is a necessary component of modern cardiac anaesthesia practice.

Another pharmacological approach to haemostasis, desmopressin, has not proven to be generally valuable. Might further studies disprove the value of antifibrinolytics, as occurred with desmospressin? Comparing the histories of these agents helps to answer this question. Desmospressin was synthesized in 1967 for the treatment of diabetes insipidis and subsequently found to shorten prolonged bleeding times. Application as a surgical haemostatic agent occurred in the following decade. The initial report in cardiac surgery demonstrated savings in blood loss, but not blood administered. ${ }^{4}$ Follow-up studies failed to demonstrate a salutary haemostatic effect of desmopressin in a variety of patient populations. ${ }^{5}$ Desmopressin's role now appears to be limited to patients who bleed and demonstrate prolongation of the bleeding time.

In contrast, the only intended use of the synthetic and natural antifibrinolytic agents was as a haemostatic agent in cardiac surgery decades ago. Initial results were not 
encouraging, probably owing to insufficient dose and administration following bypass. Epsilon aminocaproic acid (EACA) enjoyed occasional use in the ensuing years, usually as a "final" intervention for the patient bleeding excessively. Report of a successful haemostatic effect with high-dose aprotinin appeared in 1987 and with tranexamic acid in $1988 .{ }^{6,7}$ In contrast to desmopressin, confirmatory studies for these agents demonstrate consistent efficacy with prophylactic administration to diverse cardiac surgical subpopulations. Both the synthetic and natural antifibrinolytic agents may decrease bleeding by platelet preservation as well as by inhibition of fibrinolysis. ${ }^{89}$ The platelet sparing effect may be especially important since with the decreased incidence of extensive fibrinolysis defects in platelet quantity and quality are now considered the most common haemostatic defect induced by cardiopulmonary bypass. $^{10}$

The careful reader should scrutinize five specific aspects in evaluating investigations that focus on blood conservation. First, how is blood loss quantitated? Chest tube drainage measures blood loss reliably, while estimates of intraoperative blood loss are notoriously inaccurate. If antifibrinolytic drugs produce a noticeably dry field, then no such estimate can truly be blinded to the observer, thus introducing systematic bias in favour of a drug's haemostatic effect. Studies which employ only strict quantitative measurements of blood loss are preferred.

A second aspect involves the surgical team. Surgical technique can strongly influence whether drug therapy will show an effect or not. The study in which desmopressin demonstrated a significant haemostatic effect reported a large blood loss for the placebo group $(2210 \pm 1415 \mathrm{ml}){ }^{4}$ Is desmopressin of value only as an adjunct when surgical haemostasis is less meticulous? Similarly, in one study in which EACA afforded only an $18 \%$ savings in blood loss, placebo patients bled only $332 \pm 10 \mathrm{ml} .^{11}$ Might EACA provide more impressive differences had surgeons spent less time perfecting haemostasis after bypass?

Third, bleeding after cardiac surgery does not follow a classical normal distribution. Some patients bleed excessively, providing a distribution with a long "tail." Either nonparametric or logarithmically transformed analyses are appropriate for these distributions. Conclusions based on traditional analytical techniques may be erroneous.

Fourth, the reader must examine the figures for transfused blood in much the same light as the figures for blood loss. North American criteria for initiating transfusion appear more strict than European ones, the former tolerating a much lower haematocrit after surgery. European reports of large effects of aprotinin on the amount of blood transfused are not likely to be duplicated in North America. Likewise, some studies show effects on blood transfusion differences despite the placebo group receiving little blood. Those savings may become magnified in institutions with more liberal blood transfusion criteria.

Fifth, the safety aspects of antifibrinolytics should be addressed by prospective studies and not anecdotal reports. The latter create inflated incidence figures from a bias of ascertainment.

Should one embrace prophylactic antifibrinolytic therapy? The cost issue must be addressed soberly, as done by Hardy and Desroches. ' Particularly for a prophylactic regimen, one must balance the cost of therapy against the benefit of saving a half or so litre of blood. More data should be forthcoming to help the clinician choose whether and how to implement this aspect of anaesthetic care.

Finally, what is the best measure of the effectiveness of any proposed regimen to decrease bleeding following cardiopulmonary bypass? In groups of patients who start with the same haemoglobin concentration and are transfused to maintain the same target haemoglobin, the homologous blood requirements may be the best measure of effectiveness with the gold standard or ultimate goal being the elimination of all homologous blood transfusion.

\section{Hémostase réussie en chirurgie cardiaque}

Le saignement excessif à la suite de circulation extracorporelle demeure une des complications majeures à craindre en chirurgie cardiaque. L'hémostase incomplète demeure évidemment une cause de saignement mais on mentionne aussi souvent la présence de maladies concomitantes, l'interaction héparine-protamine, des anomalies quantitatives et qualitatives des plaquettes, la fibrinolyse et enfin, l'hypertension maligne. Dans ce numéro où ils passent en revue la littérature sur le sujet, Hardy et Desroches réfléchissent sur l'intérêt des agents antifibrinolytiques, naturels ou synthétiques, en chirurgie cardiaque. ${ }^{1}$ Il est admis que l'emploi de ces agents a pu réduire les pertes sanguines et dans certains cas, les besoins transfusionnels, mais il faut souligner en passant qu'ils ne sont pas les seuls et que d'autres méthodes et agents existent pour ce faire.

L'anamnèse demeure la meilleure façon de détecter une diathèse hémorragique; ainsi, la réponse hémostatique à la chirurgie antérieure et aux médications courantes (vg aspirine, antiplaquettaire à faible dose) peut suggérer de recourir à des tests supplémentaires. De plus, dans des cas majeurs où l'hémorragie représente une 
menace permanente, la connaissance préalable du profil hémostatique permet d'éliminer une pathologie préexistante si l'hémorragie devient réalité. En général, un profil normal et une anamnèse négative suffisent pour éliminer une diathèse hémorragique.

Toute opération sous circulation extracorporelle rend inévitable l'emploi de l'héparine et de son antidote habituel, la protamine. On ne peut guère compter pour changer cet état de fait sur la défibrination préopératoire avec l'ancrod; en effet, cette technique a peu de chance de devenir populaire si on considère la longue préparation préopératoire qu'elle impose et l'incontournable nécessité d'administrer du fibrinogène de source humaine après la circulation extracorporelle. Il s'ensuit donc que l'héparine circulante résiduelle après antidote et/ou des taux de protamine trop élevés continueront d'être causes de saignements excessifs postopératoires.

Bull et ses collaborateurs étudiant un très grand nombre de patients, ont montré l'existence d'une variation du simple au triple dans la sensibilité à une dose d'héparine et du simple au quadruple pour la durée d'action de cette dose; ce qui indique que la réponse d'un patient donné peut varier à l'intérieur d'un multiple de douze. ${ }^{2}$ Constatant qu'une anticoagulation tout juste à la limite durant la circulation extracorporelle peut provoquer une activation à bas bruit des mécanismes de coagulation (ce qui consomme ainsi les facteurs de coagulation et provoque une fibrinolyse secondaire), ces auteurs ont recommandé le titrage de l'activité de l'héparine pour assurer une anticoagulation sécuritaire. La remarquable diminution de l'incidence de fibrinolyse à la suite de circulation extracorporelle observée à la fin des années 70 s'explique probablement par l'anticoagulation plus satisfaisante qui a résulté de l'usage quasiuniversel de ce concept du titrage de l'activité de l'héparine. ${ }^{3}$

A quelques rares exceptions près, la protamine est le seul antidote employé pour neutraliser l'héparine. On a proposé de nombreux ratios héparine-protamine pour assurer la neutralisation de l'activité héparinique cependant, la confirmation de cette neutralisation est essentielle et c'est par titrage de la protamine elle-même qu'on peut démontrer facilement de façon concluante la présence ou l'absence d'héparine.

La prévention de la crise hypertensive ne passe pas nécessairement par l'hypertension délibérée mais plutôt par l'administration d'une anesthésie adaptée qui combine l'usage judicieux d'agents vasodilatateurs ou bétabloquants, afin de prévenir des tensions excessives sur les sutures et les effets catastrophiques qui peuvent en résulter: une anesthésie cardiaque de qualité constitue donc le premier élément de la prévention de l'hémorragie.
La desmopressine, comme appoint pharmacologique à l'hémostase, n'a pas fait la preuve de son utilité. D'autres études vont-elles réserver le sort de la desmopressine aux antifibrinolytiques? L'étude comparée de la « biographie » de ces deux agents permet de répondre à cette question. Synthétisée en 1967, la desmopressine avait été conçue pour le traitement du diabète insipide et c'est plus tard qu'on a découvert son action bénéfique sur les temps de saignement prolongés; son utilisation comme agent hémostatique en chirurgie a suivi dans la décennie suivante.

Les premiers rapports de son utilisation en chirurgie cardiaque démontraient une diminution de la perte sanguine mais aucun effet sur le nombre de transfusions administrées. ${ }^{4}$ Des études subséquentes n'ont pas réussi à démontrer l'effet hémostatique de la desmopressine dans divers groupes de patients. ${ }^{5}$ On reconnaît maintenant à la desmopressine un rôle qui se limite aux patients qui saignent et qui présentent un temps de saignement prolongé.

Au contraire, depuis des décennies, pour les agents antifibrinolytiques synthétiques et naturels, le seul usage prévu était à titre d'agent hémostatique en chirurgie cardiaque. Les premiers rapports n'étaient pas encourageants, probablement dus à l'administration de doses insuffisantes à la suite de la chirurgie extracorporelle. L'acide epsilon aminocaproique (EACA) a été sporadiquement employé dans les années suivant son introduction, habituellement comme dernier recours.

En 1987, on rapporte un effet hémostatique intéressant avec des doses élevées d'aprotinine et en 1988 avec l'acide tranexamique. ${ }^{6.7}$ Contrairement à la desmopressine, des études subséquentes confirment cet effet et démontrent leur efficacité en administration prophylactique dans des sous-groupes de patients de chirurgie cardiaque. Les antifibrinolytiques synthétiques et naturels peuvent diminuer le saignement par protection des plaquettes aussi bien que par inhibition de la fibrinolyse. ${ }^{8,9}$ L'effet protecteur sur les plaquettes peut être particulièrement important considérant qu'avec la diminution de la fibrinolyse excessive comme cause de saignement, les anomalies quantitatives et/ou qualitatives des plaquettes sont maintenant en avant-scène comme cause de saignement excessif à la suite de chirurgie extracorporelle. ${ }^{10}$

Le lecteur attentif qui veut suivre les travaux portant sur la conservation du sang devrait s'arrêter sur cinq éléments spécifiques :

Premièrement, comment la perte de sang est-elle mesurée? Le sang perdu par les drains pleuraux se mesure de façon précise alors que la perte de sang peropératoire, cela est bien connu, est jugée à l'estime (parfois fantaisiste). Si les antifibrinolytiques amènent un champ 
opératoire bien sec, il est évidemment impossible de masquer un tel état de chose à l'observateur introduisant ainsi un biais systématique en faveur de l'effet hémostatique du médicament. Le lecteur préférera les études qui s'appuient sur une mesure quantitative stricte du sang perdu.

Le deuxième élément important a trait à l'équipe chirurgicale; la technique chirurgicale peut avoir une influence majeure sur l'effet apparent de la médication. Dans l'étude où la desmopressine montrait un effet hémostatique significatif, les pertes sanguines observées dans le groupe traité au placebo étaient importantes $(2210 \pm 1415 \mathrm{ml}){ }^{4}$ La desmopressine n'a-t-elle de valeur que lorsqu'elle sert d'appoint hémostatique à une technique chirurgicale peu minutieuse? De la même façon, dans une étude où la EACA n'avait réduit les pertes sanguines que de $18 \%$, les patients du groupe placebo, pour leur part, n'avaient perdu que $332 \pm 10$ $\mathrm{ml} .{ }^{11}$ Les différences seraient-elles plus impressionnantes en faveur de EACA si les chirurgiens étaient moins minutieux à l'égard de l'hémostase après la circulation extracorporelle?

Troisièmement, les cas de chirurgie cardiaque, vus sous l'angle des pertes sanguines, ne dessinent pas une courbe de distribution normale. Certains patients saignent de façon excessive étalant ainsi la courbe de façon asymétrique; il faut analyser ces distributions de façon non paramétrique ou les transformer sous forme logarythmique car les conclusions qu'on tire des techniques analytiques traditionnelles peuvent être erronées.

Quatrièmement, le lecteur doit examiner les données chiffrées pour le sang transfusé sous le même éclairage que celles fournies pour les pertes sanguines. Les normes suivies pour commencer la transfusion sanguine apparaissent plus strictes en Amérique du Nord qu'en Europe; ainsi, on tolère ici un hématocrite beaucoup plus bas à la suite de la chirurgie. Il est douteux qu'on puisse reproduire ici en Amérique du Nord des travaux européens où l'aprotinine a réduit de façon importante la quantité de sang transfusée. De la même façon, certaines études observent des différences dans le sang transfusé même lorsque le groupe placebo en reçoit peu. De telles économies peuvent apparaître plus importantes là où les normes de transfusion sont plus libérales.

Et enfin, cinquièmement, l'innocuité des agents antifibrinolytiques doit faire l'objet d'études prospectives et non être basée sur des rapports anecdotiques; les anecdotes, en appuyant les prejugés, gonflent artificiellement les taux d'incidence.

Doit-on s'engager dans l'antifibrinolyse prophylactique ? L'aspect coût doit être étudié froidement comme le font Hardy et Desroches.' En particulier, pour un régime prophylactique, on doit prendre en compte le coût de la thérapeutique par rapport aux bénéfices que représente l'épargne d'un demi ou d'un litre de sang. Il faut attendre des données supplémentaires avant d'indiquer au clinicien comment s'engager dans cette voie, si seulement il doit le faire.

Finalement, quelle est la meilleure façon de mesurer l'efficacité de tout programme de réduction du saignement à la suite de chirurgie extracorporelle? Dans des groupes de patients avec un même taux d'hémoglobine de départ et qu'on transfuse afin de maintenir une même concentration d'hémoglobine, les besoins transfusionnels représentent la meilleure façon d'évaluer cette efficacité tout en sachant que le but ultime poursuivi est l'élimination de toute transfusion de sang homologue.

\section{References}

1 Hardy J-F, Desroches $J$. The usefulness of natural and synthetic antifibrinolytics in cardiac surgery. Can J Anaesth (In Press)

2 Bull et al. Heparin therapy during extracorporeal circulation. II. The use of a dose response curve to individualize heparin and protamine dosage. J Thorac Cardiovasc Surg $1975 ; 69: 685-9$.

3 Mammen EF, Koets AR, Washington DC, et al. Hemostasis changes during cardiopulmonary bypass. Sem Thromb Hemost 1985; 11: 281-92.

4 Salzman EW, Weinstein MJ, Weintraub RM, et al. Treatment with desmopressin acetate to reduce blood loss after cardiac surgery. N Engl J Med 1986; 314: 1402-6.

5 Horrow JC, Van Riper DF, Strong MD, Brodsky I, Parmet $J L$. The hemostatic effects of tranexamic acid and desmopressin during cardiac surgery. Circulation 1991; 84: 2063-70.

6 Royston D, Taylor KM, Bidstrup BP, et al. Effect of aprotinin on need for blood transfusion after repeat open-heart surgery. Lancet 1987; 2: 1289-91.

7 Collier W, Hlavacek J, Horrow JC, et al. Prophylactic tranexamic acid decreases blood loss after extracorporeal circulation. Anesthesiology 1988; 69: A135.

8 Soslau G, Horrow J, Brodsky I. The effect of tranexamic acid on platelet ADP during extracorporeal circulation. A J Hematol 1991; 38: 113-9.

9 van Oeveren $W$, Harder $M P$, Roozendaal $K J$, et al. Aprotinin protects platelets against the initial effect of cardiopulmonary bypass. J Thorac Cardiovasc Surg 1990; 99: 788-97.

10 Jobes $D R$, Ellison $N$. Ch. 13, Effective hemostasis in the cardiac surgical patient. In: Ellison N, Jobes DR (Eds.). Effective Hemostasis In Cardiac Surgery, WB Saunders, 1988. 195-201.

11 Vander Salm T, Ansell JE, Okike ON, et al. The role of epsilon aminocaproic acid in reducing bleeding after cardiac operation: a double-blind randomized study. J Thorac Cardiovasc Surg 1988; 95: 538-40. 